\title{
Integrative proteomic network analyses support depot-specific roles for leucine rich repeat LGI family member 3 in adipose tissues
}

\author{
HYUN A. KIM, KWANG JIN BAEK and HYE-YOUNG YUN \\ Department of Biochemistry, Chung-Ang University, College of Medicine, Seoul 06974, Republic of Korea
}

Received May 12, 2020; Accepted May 12, 2021

DOI: $10.3892 /$ etm.2021.10269

\begin{abstract}
LGI family member 3 (LGI3) is a member of the LGI protein family. In our previous studies, LGI3 was determined to be expressed in adipose tissues, skin and the brain, where it served as a pleiotropic cytokine. The results indicated that LGI3 levels are increased in adipose tissues of obese individuals in comparison with control individuals and that LGI3 suppressed adipogenesis via its receptor, disintegrin and metalloproteinase domain-containing protein 23 . Additionally, it was reported that LGI3 upregulates tumor necrosis factor- $\alpha$ and downregulated adiponectin and hypothesized that LGI3 may act as a proinflammatory adipokine involved in adipose tissue inflammation. In the present study, cytokine arrays were used to analyze cytokine levels in adipose tissues and plasma of LGI3-knockout mice and signaling protein arrays used to analyze the expression and phosphorylation of these proteins in LGI3-treated preadipocytes. The results suggested that expression levels of 129 gene products ( 24 cytokines and 105 signaling proteins) were altered in response to LGI3 deficiency or LGI3 treatment, respectively. Protein-protein interaction network analysis of LGI3-regulated gene products revealed that $94 \%$ of the gene products ( 21 cytokines and 100 signaling proteins) formed an interaction network cluster. Functional enrichment analysis for the LGI3-regulated gene products, including those from our previous studies, revealed an association with numerous biological processes, including inflammatory responses, cellular differentiation and development and metabolic regulation. Gene co-expression network analysis revealed that these LGI3-regulated gene products were involved in various biological processes in an overlapping and differential manner between subcutaneous and visceral adipose tissues. Notably, inflammatory responses were more strongly associated with the LGI3-regulated gene
\end{abstract}

Correspondence to: Dr Hye-Young Yun, Department of Biochemistry, Chung-Ang University, College of Medicine, 84 Heukseok-ro, Dongjak-gu, Seoul 06974, Republic of Korea E-mail: hyyunoffice@gmail.com

Key words: leucine-rich repeat LGI family member 3, cytokine, adipokine, adipose tissue, array, signaling, network co-expression network in visceral adipose tissues than in subcutaneous adipose tissues. Analysis of expression quantitative trait loci identified four single nucleotide variants that affect expression of LGI3 in an adipose depot-specific manner. Taken together, the results suggested that LGI3 may serve depot-specific roles as an adipokine in adipose tissues.

\section{Introduction}

Leucine-rich repeat LGI family member 3 (LGI3; formerly known as leucine-rich glioma inactivated 3) is a secretory protein belonging to the vertebrate LGI family that is abundantly expressed in the brain (1). LGI3 expression in the brain has been suggested to be regulated by activating enhancer-binding protein 2 and neuron-restrictive silencer at the transcriptional level (1). Our research group previously reported that LGI3 regulates neuronal exocytosis and differentiation $(2,3)$. Additionally, LGI3 expression in the epidermal layer of the skin has been identified, where it may act as a cutaneous cytokine (4). Our group previously established that LGI3 is secreted by keratinocytes in response to ultraviolet $\mathrm{B}$ irradiation, protecting cells (4). It was also determined that LGI3 promotes the migration, differentiation and inflammatory responses of keratinocytes (5-8) and melanocyte pigmentation (9). LGI3 may also be associated with the cytokine network in cancer (10-12) and its expression is associated with the prognosis of patients with glioma and non-small cell lung cancer $(11,13)$. Notably, the expression and genetic variations of LGI3 may serve potential prognostic roles in various types of cancer (10).

Earlier studies have indicated that LGI3 is expressed in adipose tissues in mice and that its expression is reduced during adipogenesis and increased in the adipose tissues of obese mice $(14,15)$. It has also been demonstrated that LGI3 suppresses adipogenesis via its receptor, disintegrin and metalloproteinase domain-containing protein 23 (ADAM23) (14). Moreover, LGI3 upregulates the expression of proinflammatory genes, including TNF- $\alpha$ in macrophage cells (14), and downregulates adiponectin (15). Notably, LGI3 and TNF- $\alpha$ are mutually upregulated via $\mathrm{NF}-\kappa \mathrm{B}$, suggesting their cooperative role in metabolic inflammation in obesity (16). It is hypothesized that LGI3 is a multifunctional cytokine and proinflammatory adipokine that functionally interacts with various cytokines, adipokines, chemokines and signaling proteins (12). 
To gain an insight into the functional network of LGI3 in adipose tissues, integrative analyses were performed based on protein expression and phosphorylation arrays, gene co-expression networks (GCNs), protein-protein interaction networks and expression quantitative trait loci (eQTL). In the present manuscript, evidence was presented to support the hypothesis that LGI3 has differential functions in subcutaneous adipose tissues (SATs) and visceral adipose tissues (VATs).

\section{Materials and methods}

Animals and cell culture. All animal protocols were approved by the Institutional Animal Care and Use Committee of Chung-Ang University (Seoul, Korea). All animal studies complied with the ARRIVE guidelines (17). Briefly, all animal welfare considerations were taken including daily monitoring of health and behavior and minimizing suffering and distress by practicing euthanasia as described below. LGI3-knockout mice were generated by Macrogen, Inc. (15). Adipose tissue samples at autopsy and plasma collected before euthanasia were obtained from 6 10-week-old mice (mean body weight, $26 \mathrm{~g}$ ) bred and maintained in a rodent facility under a $12 \mathrm{~h}$ light/dark cycle, at a relative humidity of $55 \pm 15 \%$ and a constant temperature $\left(23 \pm 3^{\circ} \mathrm{C}\right)$. The animals were provided with food and water ad libitum. A total of 6 male mice (three wild type and three LGI3 homozygous knockout mice) were euthanized by cervical dislocation and death was confirmed by loss of respiration and heartbeat. The duration of the experiment was 10 weeks and no mice were found dead during the period. White adipose tissues (WATs, epididymal fat) and plasma were obtained from mice and 3T3-L1 cells (American Type Culture Collection) were cultured as previously described (14).

Preparation of recombinant LGI3 and protein array analysis. Recombinant LGI3 protein was purified as previously described (3). Briefly, LGI3-His ${ }_{6}$ protein was expressed in E. coli BL21 (DE3) using the pET28a $(+)$ expression vector (Novagen; Sigma-Aldrich; Merck KGaA) and chaperone system (pGro7; Takara Bio., Inc.). The protein was purified using TALON Metal Affinity Resin (Clontech Laboratories, Inc.). 3T3-L1 cells were treated with LGI3 $(10 \mathrm{ng} / \mathrm{ml})$ at $37^{\circ} \mathrm{C}$ for 1 and $24 \mathrm{~h}$ for phosphoprotein array analysis and signaling protein analysis, respectively. Phosphoprotein array analysis was performed using the Phospho Explorer Antibody Array (cat. no. PEX100; Full Moon BioSystems, Inc.). Signaling protein analysis was performed using the Signaling Explorer Antibody Array (cat. no. SET100; Full Moon BioSystems, Inc.) and the Explorer Antibody Array (cat. no. ASB600; Full Moon BioSystems, Inc.). All assays were performed in accordance with the manufacturer's instructions. Cell extracts were prepared using the protein extraction buffer (EXB050; Full Moon BioSystems, Inc.) and Antibody Array Assay Kit (cat. no. KAS02; Full Moon BioSystems, Inc.) and analyzed using ExDEGA 1.1.9.0 (eBiogen, Inc.) according to the manufacturer's protocol. Data normalized using $\mid \log _{2}($ fold change) $\mid \geq 1$ $(\mathrm{P}<0.05)$ were used for integrative analyses.

Preparation of adipose tissues and cytokine array analysis. Adipose tissue extracts were prepared by homogenizing tissues in Dulbecco's phosphate-buffered saline (Sigma-Aldrich; Merck KGaA) containing a cocktail of protease inhibitors (Roche Diagnostics). After homogenization, Triton X-100 was added to a final concentration of $1 \%$ and the samples were frozen at $-70^{\circ} \mathrm{C}$, thawed and centrifuged at $10,000 \times \mathrm{g}$ for $5 \mathrm{~min}$ at $4^{\circ} \mathrm{C}$. The supernatants were then used to investigate the cytokine profile by employing the Mouse XL Cytokine Array kit (cat. no. ARY028; R\&D Systems, Inc.) according to the manufacturer's protocol. The Mouse XL Cytokine Array kit differs from the Adipokine and Cytokine Array Kits used in our previous study (12). These contain 111 capture antibodies, including 76 cytokine antibodies that were not included in the arrays of the previous study.

Protein-protein interaction network and functional enrichment analysis. A protein-protein interaction network was generated using data from the Search Tool for the Retrieval of Interacting Genes/Proteins (version 11.0; http://string-db. org) (18) and visualized by Cytoscape 3.8.0 (https://cytoscape. org) using an interaction degree-sorted circle layout (19). Network centrality was analyzed by Cytoscape 3.8.0 using the closeness centrality method (20). Functional enrichment analysis and Kyoto Encyclopedia of Genes and Genomes (KEGG) pathway analysis were performed using the Database for Annotation, Visualization and Integrated Discovery (version 6.8; https://david.ncifcrf.gov) (21). The results were sorted based on the $\mathrm{P}$-values and entries with $\mathrm{P}<0.05$ were presented.

Gene co-expression network (GCN) analysis. GCN analysis was performed using the GCNs of human SATs (UUID: 7054a727-5ca3-11e7-8f50-0ac135e8bacf) and VATs (omentum; UUID: c8749eba-5ca3-11e7-8f50-0ac135e8bacf) (22) obtained from the Network Data Exchange (NDEx; version 2.4.5; http://www.ndexbio.org) (23) and visualized by Cytoscape 3.8.0 using the prefuse force-directed layout. The gene ontology (GO) categories associated with the GCN were mapped using BiNGO 3.0.4 (http://apps. cytoscape.org/apps/bingo) and visualized by Cytoscape 3.8.0 using the edge-weighted spring-embedded layout. The hypergeometric test and Bonferroni correction were used to obtain P-values (24). Comparative analysis of categorized gene groups was presented as a Venn diagram generated using Venny 2.1 (http://bioinfogp.cnb.csic.es/tools/venny). The association between genes and transcription factors with respect to transcriptional regulation was assessed using transcription factor affinity prediction (http://trap.molgen.mpg. de) tools (25). RNA-seq data were obtained from the Human Protein Atlas (https://www.proteinatlas.org) and the GTEx project (https://www.gtexportal.org).

Analysis of eQTL. Single-tissue eQTL of adipose tissues were identified by searching the Genotype-Tissue Expression (GTEx) portal (https://www.gtexportal.org) (26). RNA sequencing (RNA-seq) data for the gene expression levels in subcutaneous ( $\mathrm{n}=581)$ and visceral (omentum; $\mathrm{n}=469)$ adipose tissues were used for evaluation of the effect of eQTL on LGI3 expression. Single nucleotide variants (SNVs) in eQTL that affect LGI3 expression with $\mathrm{P}<0.01$ and false discovery rate $<0.05$ were considered to be statistically significant. 
Statistical analysis. Significance was assessed using ANOVA with Bonferroni correction. The results were considered significant at $\mathrm{P}<0.05$. Statistical analyses were conducted using SPSS version 26 (IBM Corp.) and all statistical tests were two-sided. The hypergeometric test and Bonferroni correction were used to obtain P-values in BiNGO analysis and nominal P-values were generated by the linear regression model between genotype and expression in eQTL analysis.

\section{Results}

Effect of LGI3 knockout on cytokine profiles. The WATs and plasma derived from wild-type and LGI3-knockout mice were employed as samples for analyzing cytokine profiles using protein arrays (Fig. 1). The results indicated that the expression levels of various cytokines were increased or decreased in LGI3-knockout mice in comparison with WT mice (Fig. 1). The cytokines with increased expression included fibroblast growth factor 1 (FGF1), adiponectin (C1Q and collagen domain containing; ADIPOQ), chemokine (C-C motif) ligand 6 (CCL6), retinoic acid receptor responder 2 (RARRES2), insulin-like growth factor binding protein 6 (IGFBP6), periostin (POSTN), cystatin C (CST3), prolactin family 2 subfamily c member 2 (PRL2C2), complement factor D (CFD), dipeptidyl peptidase 4 (DPP4), regenerating islet-derived $3 \gamma$ (REG3G), resistin (RETN), $\alpha-2$-HS glycoprotein (AHSG), insulin-like growth factor binding protein 2 (IGFBP2), serpin family E member 1 (SERPINE1), delta-like noncanonical notch ligand 1 (DLK1) and insulin-like growth factor binding protein 1 (IGFBP1) (Fig. 1 a-f, h-p, s, v-x, $\alpha$; Table I). The cytokines with reduced levels of expression included coagulation factor III, tissue factor (F3), insulin-like growth factor binding protein 5 (IGFBP5), $\mathrm{C}-\mathrm{C}$ motif chemokine ligand 11 (CCL11), growth arrest specific 6 (GAS6), C-X-C motif chemokine ligand 5 (CXCL5), TNF superfamily member 13b (TNFSF13B) and C-C motif chemokine ligand 21 (CCL21) (Table I; Fig. 1 g, q, r, t, u, y, z). CCL6, PRL2C2 and RARRES2 were found to be increased in the WATs as well as in the plasma of LGI3-knockout mice (Fig. 1; CCL6, c, p; PRL2C2, i, x; RARRES2, d, s). Several cytokines (IGFBP5, ADIPOQ, DLK1, IGFBP1 and SERPINE1) had previously been reported to be regulated by LGI3 (Table I) (12).

Effect of LGI3 on the phosphorylation and expression of signaling proteins. LGI3 and its receptor ADAM23 have been demonstrated to be expressed predominantly in 3T3-L1 preadipocytes, with their expression declining during differentiation into adipocytes (14). Thus, LGI3 may transduce intracellular signaling in preadipocytes in an autocrine and paracrine manner. To explore the LGI3-stimulated intracellular signaling pathway, phosphoprotein array and signaling protein array analyses were performed using extracts from 3T3-L1 preadipocytes treated with the LGI3 protein (Fig. 2). Expression levels of 105 proteins were found to be increased or decreased by LGI3 in comparison with a control (Fig. 2; Tables I and SI, SII). Additionally, 15 proteins showed upregulation following LGI3 phosphorylation and 18 proteins showed downregulation following LGI3 phosphorylation (Fig. 2; Tables I and SI). KEGG pathway analysis of these gene products revealed that the proteins expressed at increased levels were
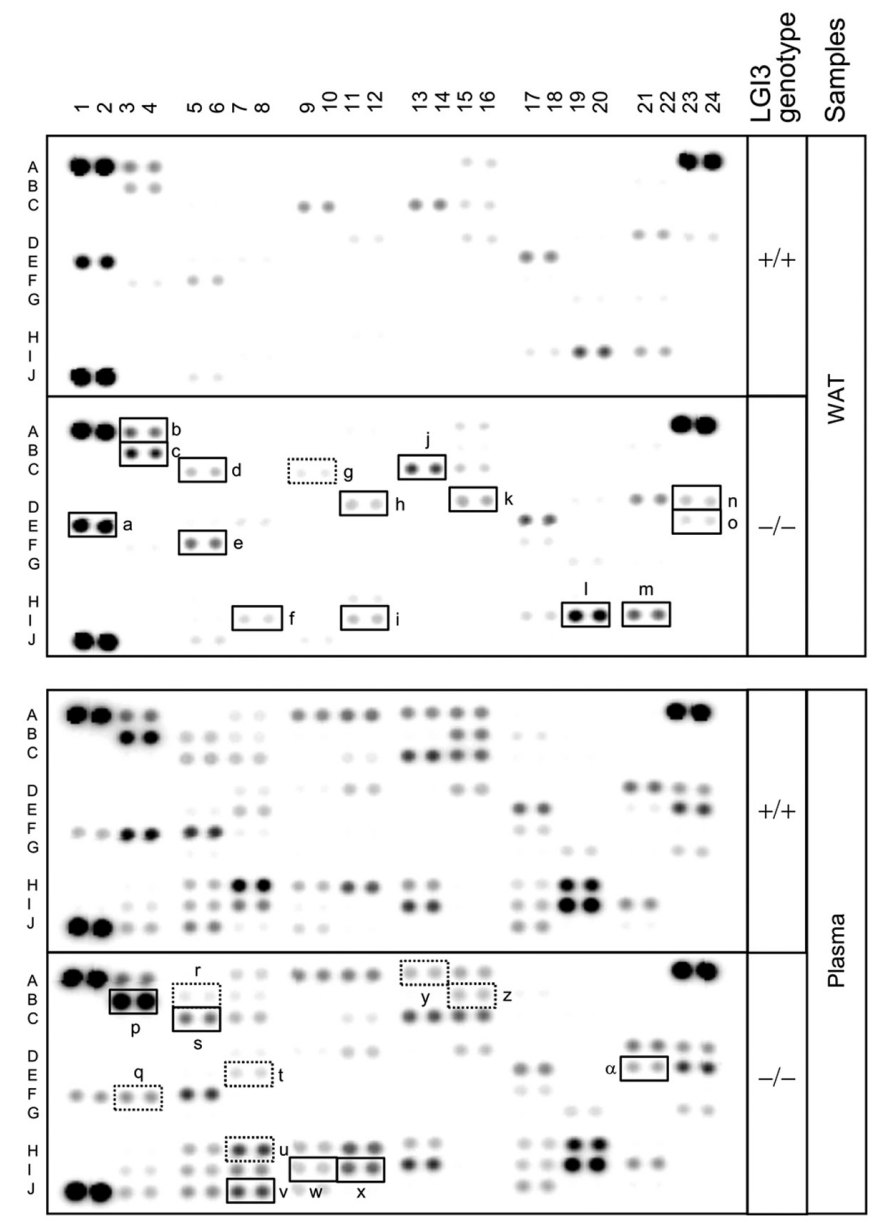

Figure 1. Effect of LGI3 knockout on cytokine profiles. WATs and plasma from wild-type (+/+) and homozygous LGI3-knockout (-/-) mice were analyzed using cytokine XL arrays. Solid-line box, increased proteins in knockout mice. Numbers and capital letters represent the labels of array coordinates (Mouse XL Cytokine Array ARY028, R\&D Systems, Inc., https://www.rndsystems. $\mathrm{com} /$ products/proteome-profiler-mouse-xl-cytokine-array_ary028). Array coordinate is listed in Table SII. Dotted-line box, decreased proteins in knockout mice. The list of proteins has been described in the Results and in Tables I and SI. a, FGF1; b, ADIPOQ; c and p, CCL6; d and s, RARRES2; e, IGFBP6; f, POSTN; g, F3; h, CST3; I and x, PRL2C2; j, CFD; k, DPP4; 1, REG3G; LGI3, leucine-rich repeat LGI family member 3; m, RETN; n, AHSG; o, IGFBP2; q, IGFBP5; r, CCL11; t, GAS6; u, CXCL5; v, SERPINE1; w, DLK1; WATs, White adipose tissues; y, TNFSF13B; z, CCL21; $\alpha$, IGFBP1.

associated with the terms 'neurotrophin signaling pathway', 'osteoclast differentiation', 'B cell receptor signaling pathway', 'PI3K/Akt signaling pathway', 'NF- $\mathrm{BB}$ signaling pathway', 'TNF signaling pathway', 'insulin resistance' and additional terms related to various infectious diseases and cancer-related pathways (Table SIII). The gene products with reduced expression levels were found to be associated with the terms 'PI3K/Akt signaling pathway', 'mTOR signaling pathway' and various cancer-related pathways (Table SIII). Signaling protein array analysis demonstrated that the expression of 27 proteins was increased, while that of 45 proteins was reduced (Fig. 2; Tables I and SI). Functional enrichment analysis of these gene products revealed that the upregulated gene products were involved in tyrosine kinase signaling pathways, cell proliferation, PI3K signaling pathway, and growth hormone receptor and melanosome functions (Table SIV). The downregulated gene products were associated with transcriptional 
Table I. Summary of LGI3-regulated gene products identified in the present study and in previous studies.

\begin{tabular}{|c|c|c|}
\hline Array & Upregulated gene products (reference no.) & Downregulated gene products (reference no.) \\
\hline Cytokine XL Array & $\begin{array}{l}\text { CCL21, CXCL5, F3, IGFBP5 }{ }^{\mathrm{d}}, \mathrm{TNFSF}^{13 B} \text {, } \\
\text { CCL11, GAS6 }\end{array}$ & $\begin{array}{l}\text { ADIPOQ }^{\mathrm{d}}, \text { AHSG, CCL6, CFD, CST3, DLK1 }{ }^{\mathrm{d}} \text {, } \\
\text { DPP4, FGF1, IGFBP1 }{ }^{\mathrm{d}}, \text { IGFBP2, IGFBP6, POSTN, } \text {, } \\
\text { PRL2C2, RARRES2, REG3G, RETN, SERPINE1 }\end{array}$ \\
\hline Phospho Explorer & BLNK, BRCA1, BTK, CALM1, CREB1, & ALK, CAV1, CBL, CD5, DOK1, ESR1, HSP90AB1, \\
\hline Antibody Array ${ }^{\mathrm{b}}$ & $\begin{array}{l}\text { ERBB2, FOXO3, GRK2, IKBKB, IL2RA, } \\
\text { JUN, LIMK1, RELA, RPS6KA1, TP73 }\end{array}$ & $\begin{array}{l}\text { IRS1, KIT, KRT18, MAPK14, MTOR, PDGFRA, } \\
\text { PRKCA, PRKCD, RYR2, STMN1, ZAP70 }\end{array}$ \\
\hline Signaling Explorer & ADCK2, AFP, CASP1, CD37, CD80, ERBB3, & ADCK1, AKT2, AXL, C1S, CAMKV, CD247, \\
\hline Antibody Array & ERN1, F10, F12, GAD1, GH1, GPR151, & CD3E, CFB, COL4A3, CRYAB, CYP2S1, \\
\hline CYP39A1, & $\begin{array}{l}\text { LYN, MSTN, PTGS1, PTK6, RCBTB1, RPS27, } \\
\text { SND1, TUBB3, TYK2, TYRO3 }\end{array}$ & $\begin{array}{l}\text { DCC, DDX4, DNAL4, EEF1G, EGF, EPHB1, EPN3, } \\
\text { EXOG, F2R, FLI1, FN1, FOXA2, KAT8, KDR, } \\
\text { KLK3, LAMC3, MATK, MUC16, NCR1, } \\
\text { NEUROG3, NFKBIA, POLR3D, POU3F1, PRPF19, } \\
\text { RCHY1, SLU7, SNAI2, TAF4, TBP, TP63, USP13 }\end{array}$ \\
\hline Explorer Antibody & CD63, E2F2, MLH1, SEMA4D, SLC3A2 & INSR, MAP2K2 \\
\hline
\end{tabular}

Array

Previously reported

AKT1 (3), CCL12 (12), CCL2 (16), CD68 (12),
CSF3 (12), CTNNB1 (5), CXCL13 (12),
CXCL2 (12), CYBA (12), CYBB (12),
EMR1 (12), FLG(6), IGF1 (12), IGFBP5 (12),
IL6 (12), ITGAX (12), IVL (6), KRT10 (6),
LOR (6), MAPK1 (12), MAPK3 (12),
MDM2 (4), MITF (9), NCF1 (12), NCF2 (12),
NFKB1 (16), NOS2 (14), PIK3CA (3),
PRKAA1 (12), PTEN (12), PTGS2 (14),
PTK2 (3), TGM1 (6), TIMP1 (12), TNF (14,16)

ADIPOQ (15), BAD (12), C5 (12), CEBPA (14), CRP (12), CSF1 (12), DLK1 (12), EIF4EBP1 (12), ESM1 (12), FABP4 (14), GSK3A (12), GSK3B (5), IGFBP1 (12), LPL (14), PPARG (14), SERPINE1 (12), $\operatorname{STX1A}^{\mathrm{c}}(2), \mathrm{TP53}(4)$

\begin{abstract}
${ }^{a}$ Upregulated proteins in LGI3-knockout mice were presumed to be downregulated by LGI3, and vice versa for downregulated proteins

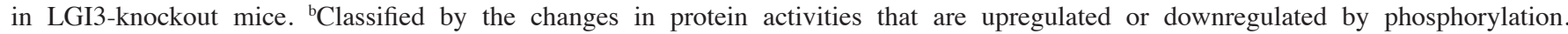

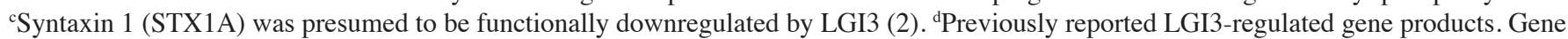
product names are the official gene symbols. The official full names of the gene symbols are listed in Table SI.
\end{abstract}

regulation, PI3K/Akt signaling pathway, $\mathrm{T}$ cell receptor signaling pathway, focal adhesion, and various cancer-related pathways (Table SIV).

Protein-protein interaction network of LGI3-regulated gene products. A protein-protein interaction network of LGI3-regulated gene products, based on both the findings of the present study (Tables I and SI) and those of our previous study (12), was constructed and visualized based on the interaction degree (Fig. 3). In total, 169 gene products $(95 \%$ of 177 LGI3-regulated gene products) were associated with the protein-protein interaction network cluster; 81 upregulated gene products and 88 downregulated gene products were involved in the interaction network cluster; and 42 gene products in the cluster were cytokines, adipokines, and chemokines (Fig. 3; inner circular subnetwork). Of the gene products, 94\% (21 cytokines and 100 signaling proteins) were identified and included the interaction network cluster, and $6 \%$ of the gene products were not associated with the network cluster. Notably, all cytokines, adipokines, and chemokines that were regulated by LGI3, with the exception of REG3G, CCL6 and PRL2C2, were included in the interaction network cluster (Fig. 3, inner circular subnetwork). Transcriptional regulatory association analysis of this subnetwork indicated that the member genes may be regulated by NF- $\kappa \mathrm{B}, \mathrm{C} / \mathrm{EBP} \alpha, \mathrm{Pax}-8$, TFIIA- $\alpha / \beta / \gamma$ and TBP $(\mathrm{P}<0.01$; Table SV). A total of 1,906 interactions were found in the LGI3-regulated protein-protein interaction network. The gene products with the highest interaction degrees (>50) included IL6, AKT1, TP53, EGF, TNF, MAPK3, FN1, MAPK1, IGF1, PTEN, JUN, CCL2, MTOR, CTNNB1, IRS1, MAPK14 and ERBB2. The cytokines, adipokines and chemokines in the network with the highest interaction degrees (>30) included IL6, EGF, TNF, IGF1, CCL2, CRP, TIMP1, SERPINE1, ADIPOQ, CSF1 and CSF3.

Functional enrichment analysis of LGI3-regulated genes. To elucidate the biological functions associated with the protein-protein interaction network of LGI3-regulated gene products, a functional enrichment analysis was performed using the gene members of the network. GO categories were mapped using statistically overrepresented functional themes in a hierarchical manner (Fig. 4). GO terms with the highest significance were associated with inflammatory responses, hormonal responses, epithelial differentiation and 


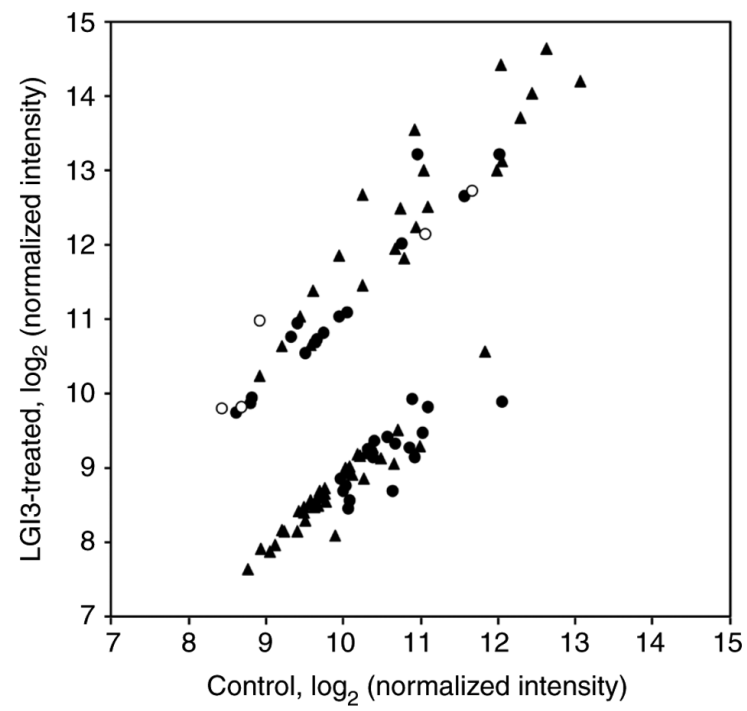

Figure 2. Effect of LGI3 on the phosphorylation and expression of signaling proteins. Scatter plots of $\log _{2}$ normalized intensities. Black circle, phosphorylation data from Phospho Explorer Antibody Array (PEX100). Triangle, data from Signaling Explorer Antibody Array (SET100). White circle, data from Explorer Antibody Array (ASB600). Data with $\mid \log _{2}$ (fold change) $\mid \geq 1$ are indicated.

development, protein phosphorylation and signaling, metabolic and transcriptional regulation, programmed cell death, and protein transport (Fig. 4). Notably, inflammatory response was the most significant and highly represented GO category.

Gene co-expression network analysis. All LGI3-regulated gene products were queried against the GCNs of SATs and VATs. GCNs are networks of genes connected by significant co-expression relationships that provide insights into the tissue-specific functions of gene sets, as co-expressed genes are regulated by common transcriptional regulatory programs and are components of the same protein complex or signaling pathway (27). A total of 121 and 114 gene products in the LGI3-regulated gene set were identified in the GCNs of SATs and VATs, respectively (Fig. 5A, group a; Fig. 5B, group c; Table SVI). These LGI3-regulated gene product subsets were associated with 5,904 gene products in the SAT GCN and with 3,743 gene products in the VAT GCN (Fig. 5A, group b; Fig. 5B, group d; Table SVI). Notably, these subnetworks of first-neighboring co-expressed genes (Fig. 5A, group b; Fig. 5B, group d) revealed distinct distributions between the GCNs of SATs and VATs. Comparative analysis was performed with the sum of the sets for LGI3-regulated genes and their first-neighboring co-expressed genes in the GCNs of SATs and VATs (Fig. 6A). The intersection of the sets included 2,669 genes, with 3,356 genes and 1,188 genes occurring only in the GCNs of SATs and VATs, respectively (Fig. 6A). A GO category map of the sum of gene co-expression subnetworks (Fig. 5, groups a-d; Fig. 6A) demonstrated that the gene products were involved in inflammatory and immune system, metabolic processes, apoptosis and vascular development (Fig. 6B). It was also observed that inflammatory response is a predominant GO term in the LGI3-regulated GCNs of VATs and that apoptosis and vascular development are preferentially associated with the LGI3-regulated GCNs of SATs (Fig. 6B; Table SVII). Transcriptional regulatory association analysis of these subnetworks predicted that distinct and common transcription factors may be involved in the LGI3-regulated GCNs of SATs and VATs (Table SVIII).

Venn diagram analysis of LGI3-regulated genes and their associated GCNs of adipose tissues revealed that six LGI3-upregulated genes and 10 LGI3-downregulated genes belonged to the set of SAT GCN, and that five LGI3-upregulated genes and four LGI3-downregulated genes belonged to the set of VAT GCN (Fig. 7A). Protein-protein interaction network analysis of the 25 LGI3-regulated genes associated with adipose tissue GCNs demonstrated that 21 gene products formed an interaction network cluster (Fig. 7B). The interaction network cluster included 14 SAT-specific and seven VAT-specific LGI3-regulated genes in GCNs (Fig. 7B). Functional enrichment analysis of these genes revealed their involvement in various biological processes in a depot-specific manner (Table II). Notably, hematopoietic cell lineage, immune response and cytokine-cytokine receptor interaction were predominant functions of LGI3-regulated genes in VAT GCN.

In the present study, GCN analysis of the LGI3-regulated gene products demonstrated distinct co-expression network profiles between SATs and VATs. RNA-seq data of adipose tissues from the Human Protein Atlas indicated that LGI3 transcripts were expressed in adipocytes [40-60\% of the total transcripts per million (TPM), fibroblasts (20-40\%), smooth muscle cells (15\%) and other cell types (5\%)]. Moreover, RNA-seq data from the GTEx project of human adipose tissues revealed that the expression of LGI3 was higher in SATs [average protein transcripts per million $(\mathrm{pTPM})=0.5$ ] than in VATs (average pTPM=0.2). The gene products in the LGI3-regulated gene co-expression subnetworks may be regulated by common transcriptional regulatory programs.

Expression quantitative trait loci for LGI3 expression in adipose tissues. To explore the adipose depot-specific regulation of LGI3, single tissue eQTL were analyzed for the genomic loci that affect the expression levels of LGI3 in adipose tissues. eQTL are SNVs in genomic loci that account for variation in expression levels of mRNA (26). It was identified that three SNVs (Fig. 8A-D) in SAT and one in VAT (Fig. 8A and E) significantly altered LGI3 (Table III). These SNVs were cis-eQTL located 0.2-1.1 Mb apart from the LGI3 gene on chromosome 8 . Analysis of single tissue RNA-seq data revealed that two SNVs (chr8_23149508_G_A_b38 and chr8_23149665_G_A_b38) increased LGI3 expression in SAT (Fig. 8C and D), and two SNVs (chr8_21801331_T_G_b38 and chr8_22361155_C_T_b38) decreased LGI3 expression in SAT and VAT (Fig. 8B and E).

\section{Discussion}

The proinflammatory adipokine hypothesis for LGI3 is supported by the increased levels of LGI3 in adipose tissues in conditions of genetic obesity or high-fat-diet-induced obesity $(14,15)$. Furthermore, our previous results revealed that LGI3 downregulated adiponectin and upregulated TNF- $\alpha(15,16)$. These results led to the hypothesis that LGI3 serves a regulatory role in the cytokine network of adipose tissues. It was further determined that LGI3-regulated gene products formed a protein-protein interaction network 


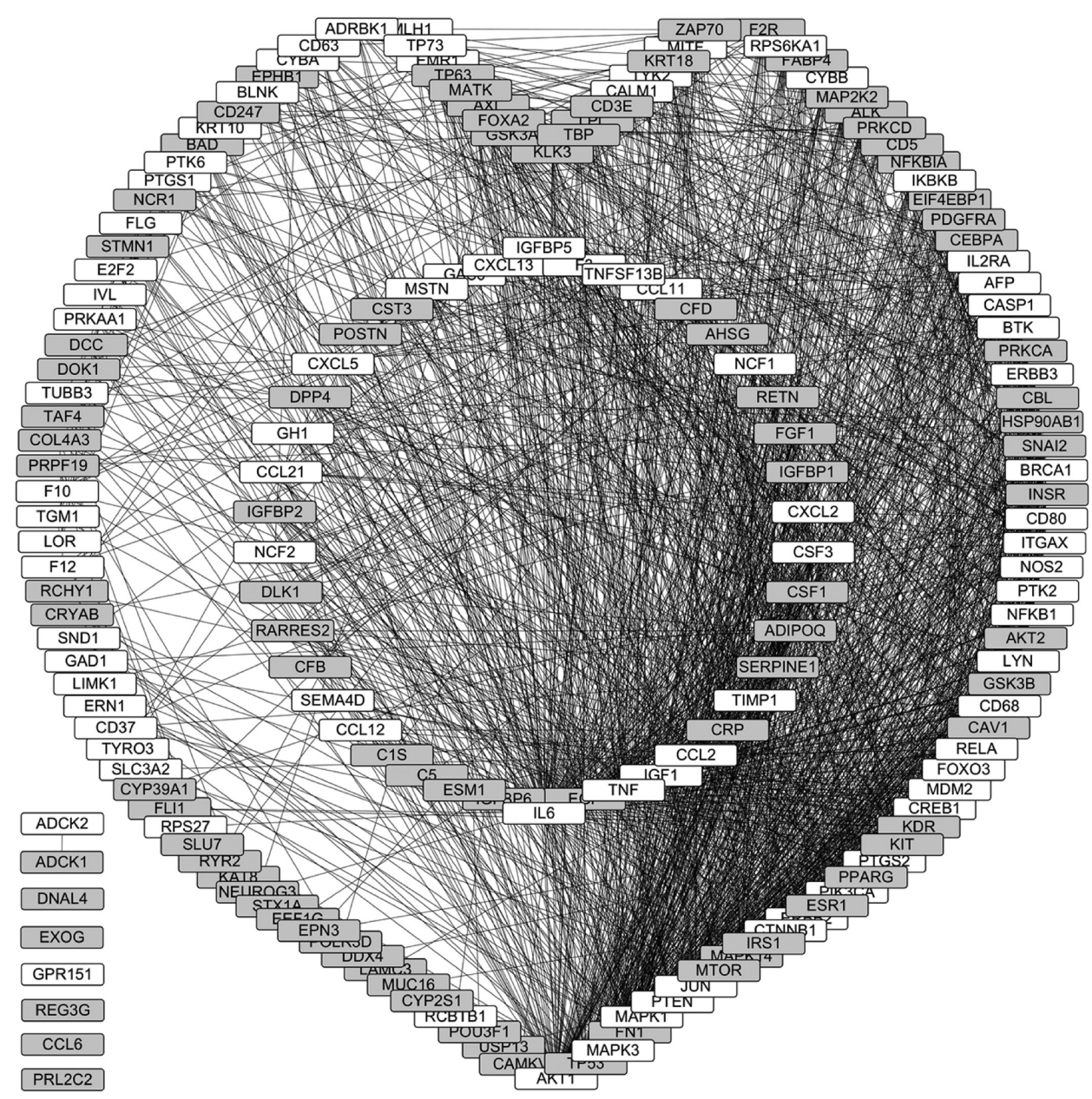

Figure 3. Protein-protein interaction network of leucine-rich repeat LGI family member 3-regulated gene products. The network is represented by nodes (box, gene products) and lines (pairwise protein-protein interactions). The nodes are sorted based on the interaction degree. The white nodes indicate the upregulated gene products, whereas the gray nodes indicate the downregulated gene products. The inner circular network indicates a subnetwork consisting of cytokines, adipokines and chemokines.

cluster (12). The present study revealed an extended LGI3-regulated protein-protein interaction network and depot-specific association of LGI3 with adipose tissues by employing GCN and eQTL analyses in addition to functional enrichment analysis used in the previous study (12). In addition to adipose tissues, LGI3-regulated protein-protein interaction networks have been implicated in the prognosis of patients with glioma (11) and non-small cell lung cancer (13). Thus, LGI3-regulated functional networks may be large with numerous gene product members in multiple tissues.

The altered expression of various cytokines in the adipose tissue and plasma of LGI3-knockout mice suggests that the regulatory interactions of LGI3 with these factors may have both local and systemic effects (12). Moreover, the increased expression of cytokines in LGI3-knockout mice may be due to compensatory upregulation in response to LGI3 deficiency or may represent cytokines that are negatively regulated by LGI3 in wild-type mice (12). The downregulated cytokines in LGI3-knockout mice suggest that LGI3 may upregulate these factors in wild-type mice (12). Moreover, the protein-protein interaction network cluster of LGI3-regulated cytokines supports the critical role of LGI3 in the homeostasis of adipose tissues through the cytokine network. It has been predicted that the LGI3-regulated cytokine network is primarily regulated by NF- $\kappa \mathrm{B}$, a key transcription factor in immune and inflammatory processes (28). It has also been demonstrated that LGI3 is a target gene and activator of NF- $\mathrm{BB}(8,16)$. Multiple genes with altered expressions in obesity have been found to be regulated by NF- $\mathrm{KB}$ (29). Thus, the increased expression of LGI3 observed in obesity may perturb the cytokine network largely through NF- $\mathrm{KB}$ and lead to metabolic inflammation in obese adipose tissues.

The components of the intracellular signaling pathways induced by LGI3 have been explored in various cell types. In neuronal cells, it has been revealed that LGI3-induced neurite outgrowth is mediated by Akt and focal adhesion kinase (3). p53 and MDM2 have also been determined to be involved in LGI3-promoted survival in ultraviolet B-irradiated keratinocytes (4). Moreover, it has been demonstrated that GSK $3 \beta$ and $\beta$-catenin mediated LGI3-promoted keratinocyte migration (5). Multiple signaling proteins (Erk1/2, AMPK, Bad, PTEN, 4E-BP1, Akt and GSK3 $\beta$ ) are regulated in LGI3-treated preadipocytes (12). Notably, the suppressive effect of LGI3 on adipogenesis in 3T3-L1 cells is mediated by its receptor, ADAM23 (14), whereas ADAM22 was determined to be the primary receptor for LGI3 in keratinocyte inflammatory signaling (8). Thus, LGI3 may transduce intracellular signaling through distinct receptors with common or unique 


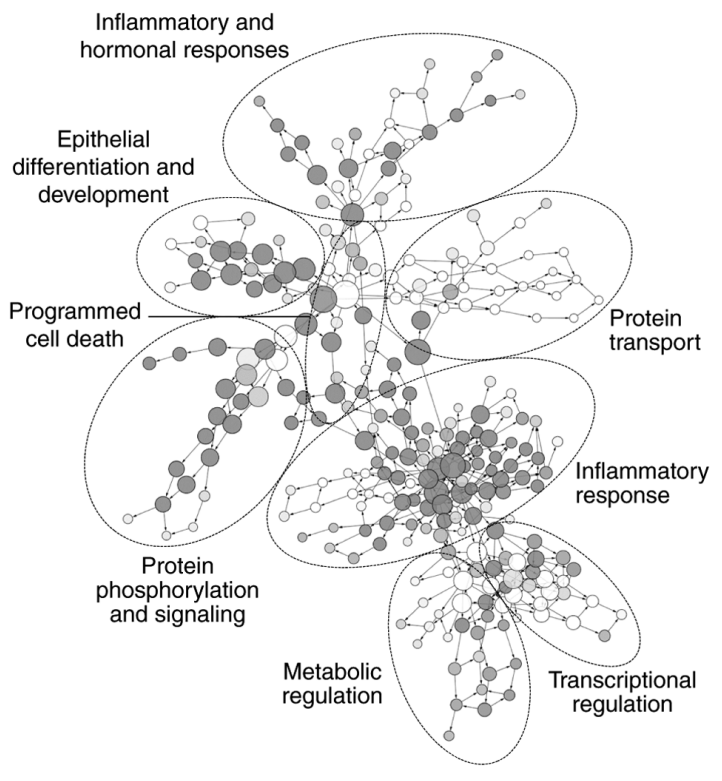

Figure 4. Functional enrichment analysis map of leucine-rich repeat LGI family member 3-regulated gene products. The nodes represent GO terms, and the arrow lines indicate parental relationships in the network hierarchy. The darker nodes represent lower P-values in the BiNGO analysis and indicate statistically more overrepresented GO terms $(\mathrm{P}<0.01)$. The dotted circles indicate the groups of GO category names that represent the GO term clusters. GO, Gene Ontology.

signaling components in various target cell types. As 3T3-L1 cells express ADAM23 but not ADAM22, the LGI3-regulated signaling protein network of the present study may represent the components and crosstalk effectors of the LGI3-ADAM23 signaling pathways.

The proximal signaling mechanisms of ADAM23 are still largely unknown. However, ADAM23 associates with $\alpha v \beta 3$ integrin to promote cell adhesion (30), with $\alpha v \beta 3$ integrin regulating macrophage inflammatory responses via PI3K/Akt-dependent NF- $\kappa$ B activation (31). Presumably, short-term upregulation by LGI3-induced protein phosphorylation occurred in the gene products that were predicted to be involved in immune response, inflammatory response and the PI3K/Akt signaling pathway, whereas gene products downregulated in response to phosphorylation were predominantly associated with cancer-related pathways $(11,13,15,16)$. These results support the hypothesis that LGI3 is a proinflammatory adipokine upregulated in conditions of obesity-associated metabolic inflammation and downregulated in cancer $(10,11,13-15)$. In the current study, long-term upregulation of expression in LGI3-treated 3T3-L1 cells was demonstrated in the gene products predicted to be associated with receptor protein tyrosine kinase/non-membrane spanning protein tyrosine kinase signaling pathway, cell proliferation, PI3K signaling pathway and growth hormone receptor function. Notably, gene products with downregulated expression were predicted to be involved in the positive regulation of transcription, cancer pathways, nuclear chromatin function, and cell migration. It was also observed that the PI3K signaling pathway and cancer-related pathways were regulated by phosphorylation and expression levels. Presumably, the suppressive effect of LGI3 on adipogenesis (14) may be mediated by ADAM23 via NF- $\mathrm{NB}$ and $\mathrm{PI} 3 \mathrm{~K} / \mathrm{Akt}$ pathways. $\mathrm{NF}-\kappa \mathrm{B}$ may be activated cooperatively
A
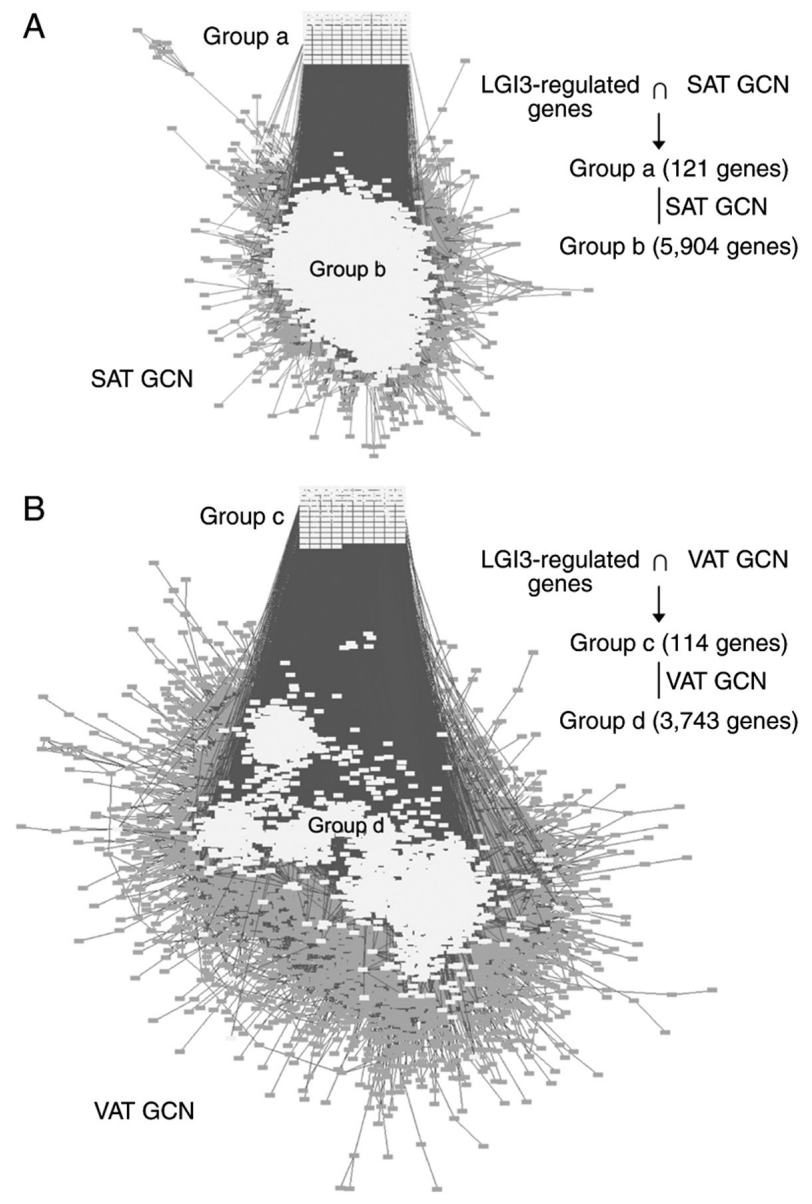

Figure 5. GCN analysis of LGI3-regulated gene products in adipose tissues. (A) LGI3-regulated gene products associated with the co-expression subnetwork of SAT GCN (group a) and a group of their first-neighboring co-expressed genes of SAT GCN (group b). (B) LGI3-regulated gene products associated with the co-expression subnetwork of VAT GCN (group c) and a group of their first-neighboring co-expressed genes of VAT GCN (group d) GCN, gene co-expression network; LGI3, leucine-rich repeat LGI family member 3; SAT, subcutaneous adipose tissue; VAT, visceral adipose tissue.

by LGI3 and TNF- $\alpha$ to antagonize insulin signaling in adipogenesis (14). LGI3 may also regulate the PI3K/Akt pathway, a component of adipogenic signaling pathways, by an unknown mechanism that may counteract adipogenesis.

The majority of LGI3-regulated gene products were involved in a protein-protein interaction network cluster with high interaction degrees. In the present study, the LGI3-regulated interaction network reported in our previous study (12) was extended by $\sim 4$ fold in node number. Statistical analysis of network centrality to identify critical gene products in the network revealed that the gene products with the highest centrality in rank order were AKT1, IL6, TP53, EGF, TNF, FN1, MAPK3, MAPK1, PTEN, IGF1, JUN, CCL2, MTOR, CTNNB1, MAPK14, ESR1, IRS1, ERBB2, PIK3CA and PPARG (data not shown). These gene products have previously been reported to be involved in obesity-associated metabolic disorders (32-51). Moreover, a subset of these gene products has been previously reported to be regulated by LGI3; these included AKT1, TP53, TNF, MAPK3, MAPK1, PTEN, IGF1, CTNNB1, PIK3CA and PPARG $(4-6,12,16)$.

The functional enrichment map of LGI3-regulated gene products demonstrated that the LGI3-regulated protein-protein 
A

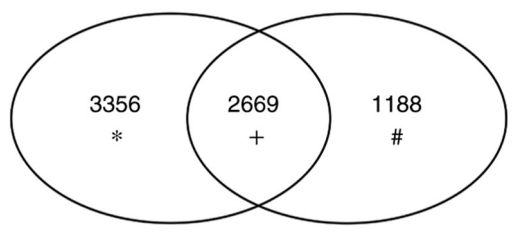

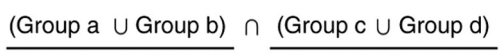

B

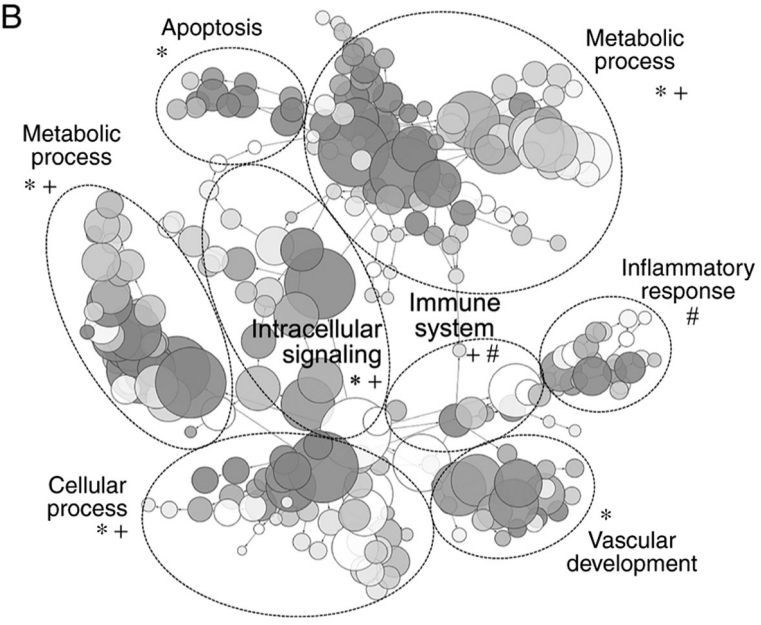

Figure 6. Comparative and functional enrichment analyses of the gene co-expression subnetworks of LGI3-regulated gene products and their first-neighboring co-expressed genes in adipose tissue GCNs. (A) Venn diagram indicating the distribution of genes in LGI3-regulated SAT and VAT GCNs. (B) Functional enrichment analysis map of LGI3-regulated SAT and VAT GCNs. The nodes represent GO terms and the arrow lines indicate parental relationships in the network hierarchy. The darker nodes represent lower $\mathrm{P}$-values $(\mathrm{P}<0.01)$. The dotted circles indicate the groups of GO category names that represent the GO term clusters. Differences (* and \#) and intersections (+) of gene sets (Fig. 6A) in LGI3-regulated GCNs are indicated. GCN, gene co-expression network; LGI3, leucine-rich repeat LGI family member 3; SAT, subcutaneous adipose tissue; VAT, visceral adipose tissue.

interaction network was associated most significantly with inflammatory responses, epithelial differentiation and development, and metabolic regulation. Previous results indicated that LGI3 may serve a proinflammatory role by upregulating COX-2, iNOS, MCP-1, TNF- $\alpha$ and NF- $\mathrm{BB}(14,16)$. Notably, LGI3 differentially increases the expression of multiple inflammatory genes in preadipocytes, adipocytes and macrophages (12). These LGI3-regulated inflammatory gene products are known to be involved in obesity-associated metabolic disorders $(36,52-61)$. It was also previously reported that LGI3 regulated the differentiation of keratinocytes (6), neuronal cells (3) and adipocytes (14), as well as the inflammatory response of keratinocytes (8), preadipocytes, adipocytes and macrophages (12). These results support the multitarget and pleiotropic nature of LGI3.

Comparative analysis of the LGI3-regulated gene products in the GCNs of SATs and VATs revealed common and distinct subsets of gene products. A larger number of gene products were involved in the LGI3-regulated GCNs in SATs than in VATs. The larger size of the LGI3-regulated SAT GCN may be due to the relatively higher expression of LGI3 in SATs than in VATs, and the difference in the transcription factor repertoires that control LGI3-regulated GCNs. The functional enrichment map of LGI3-regulated GCNs of SATs
A

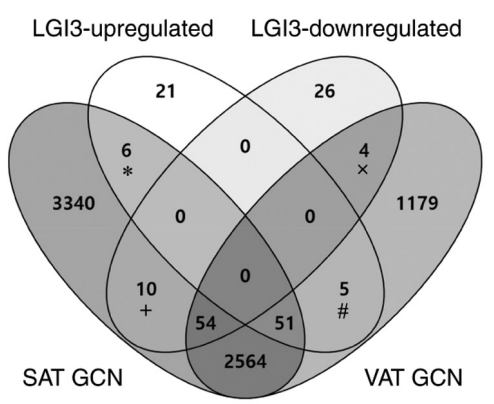

B

SAT

VAT

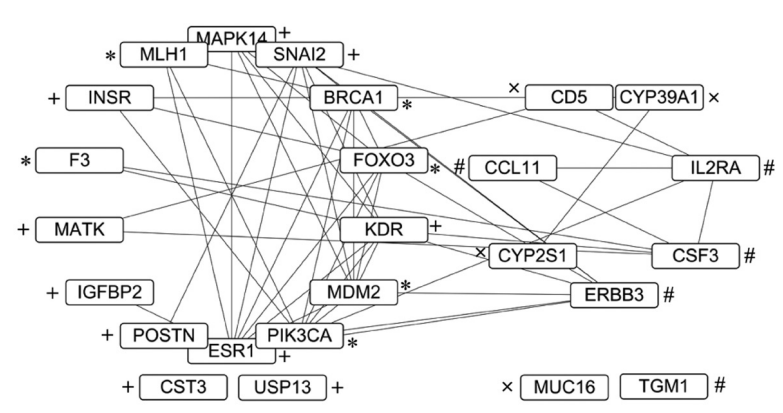

Figure 7. Comparative analysis of the upregulated or downregulated genes by LGI3 and the gene sets of adipose tissue GCNs associated with LGI3-regulated genes. (A) Venn diagram presenting the sets of regulated gene categories. (B) Protein-protein interaction network of LGI3-regulated genes in adipose tissue GCNs. The network is depicted by nodes (gene products) and lines (pairwise protein interactions) sorted based on interaction degrees. The symbols $(*, x,+$ and \#) indicate the gene products in the common sets of the gene categories indicated in Fig. 7A. GCN, gene co-expression network; LGI3, leucine-rich repeat LGI family member 3; SAT, subcutaneous adipose tissue; VAT, visceral adipose tissue.

and VATs revealed that vascular development and inflammatory responses are the predominant function categories in SATs and VATs, respectively. These results imply that LGI3 may serve as a multifunctional cytokine in cellular differentiation and development $(3,6,12)$ in SATs and as a proinflammatory adipokine in obesity-associated metabolic disorders in VATs (14-16). GCN analysis of the metabolic disorder-associated modules of the co-expressed genes in adipose tissues demonstrated enrichment of the immune response and oxidative phosphorylation pathways (62). Moreover, GCN and protein interaction network analyses indicated that NF- $\mathrm{KB}$ was implicated in angiogenesis and inflammation in adipose tissue (29). Transcription factor GCN of adipose tissue RNA-seq data also indicated that the obesity-associated network module was enriched for regulation processes in the immune system (63). Protein-protein interaction networks of LGI3-regulated gene products associated with adipose tissue GCNs further supported that these gene products may be differentially regulated in SAT and VAT in a cooperative manner. Hence, LGI3-regulated GCNs may serve multiple and differential roles in the homeostasis and dysregulation of metabolic, cellular and inflammatory processes in SATs and VATs.

Whole-genome analysis of eQTL revealed four SNVs that account for the regulation of LGI3 expression in adipose tissues. All eQTL were located within $\sim 1.1 \mathrm{Mb}$ of the LGI3 gene and may serve as a cis-acting regulatory elements for LGI3 expression. These eQTL were located in intergenic regions 
A

Chromosome 8

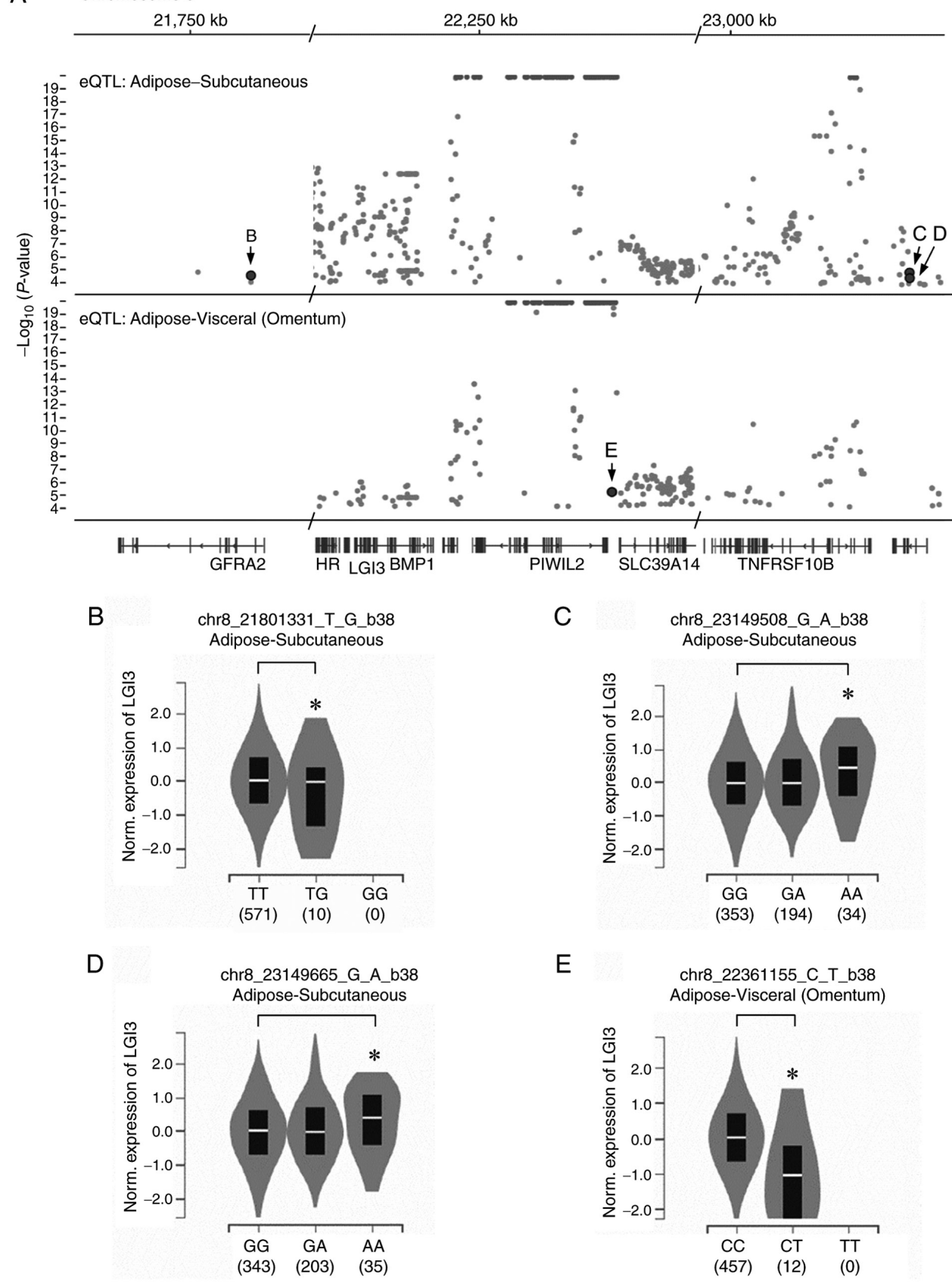

Figure 8. eQTL that affect the expression of LGI3 in adipose tissues. (A) Single-tissue eQTL tracks. Each dot in the scatter plots represents a significant eQTL (FDR <0.05). Large dots (B-E) indicate significant eQTL for LGI3. Slash lines in horizontal axis indicate the regions of chromosome omitted in the diagram to show widespread eQTLs collectively. (B-E) eQTL violin plots for significant eQTL that affect LGI3 expression in a depot-specific manner. The X-axis is the variant alleles and sample numbers and the $\mathrm{y}$-axis is normalized expression values of LGI3. The eQTL for LGI3 are summarized in Table III. Variant IDs of eQTL are as follows: (B) chr8_21801331_T_G_b38; (C) chr8_23149508_G_A_b38; (D) chr8_23149665_G_A_b38; and (E) chr8_22361155_C_T_b38. ${ }^{*}$ FDR $<0.05$ and $\mathrm{P}<0.01$. eQTL, expression quantitative trait loci; LGI3, leucine-rich repeat LGI family member 3; Norm, normal.

(chr8_21801331_T_G_b38, chr8_22361155_C_T_b38) and in introns of the TNFRSF10D gene (chr8_23149508_G_A_b38, chr8_23149665_G_A_b38). Notably, an intergenic SNV (chr8_22361155_C_T_b38) decreased the expression of LGI3 in VAT. These results suggested that genetic variations in SNVs near the LGI3 gene are associated with adipose depot-specific regulation of LGI3.

Macrophage polarization in adipose tissues is a hallmark of the proinflammatory switch in obesity (64-66), and the predominance of proinflammatory M1-type macrophages over those of the anti-inflammatory M2-type represents a major component of metabolic inflammation in VATs $(67,68)$. Our previous studies indicated that LGI3 upregulated M1-polarized macrophage markers (TNF- $\alpha$, iNOS, CCL-2/MCP-1, IL-6, CD68 and CD80) $(12,14,16)$. The increased expression of LGI3 in adipose tissue macrophages is observed in obesity and may serve a critical regulatory role in M1 macrophage polarization and proinflammatory transition of cytokine networks $(12,14,16)$. It was hypothesized that LGI3 may contribute to innate tumor immunity in the cancer microenvironment by promoting M1 polarization of the tumor-associated macrophages (13). Thus, LGI3-regulated cytokine networks may serve pathological 
Table II. Functional enrichment analysis of the LGI family member 3-regulated gene products associated with GCNs of SAT and VAT.

\begin{tabular}{|c|c|c|c|}
\hline Category & Term & Count & P-value \\
\hline GO TERM_MF_DIRECT & GO:0005515 protein binding & 16 & $5.53 \times 10^{-5}$ \\
\hline KEGG_PATHWAY & hsa04068:FoxO signaling pathway & 5 & $6.04 \times 10^{-5}$ \\
\hline GO TERM_BP_DIRECT & GO:0032355 response to estradiol & 4 & $6.68 \times 10^{-5}$ \\
\hline KEGG_PATHWAY & hsa04151:PI3K-Akt signaling pathway & 6 & $1.82 \times 10^{-4}$ \\
\hline KEGG_PATHWAY & hsa04917:Prolactin signaling pathway & 4 & $2.17 \times 10^{-4}$ \\
\hline KEGG_PATHWAY & hsa05205:Proteoglycans in cancer & 5 & $2.86 \times 10^{-4}$ \\
\hline GO TERM_BP_DIRECT & GO:0090090 negative regulation of canonical Wnt signaling pathway & 4 & $3.75 \times 10^{-4}$ \\
\hline GO TERM_BP_DIRECT & GO:0030335 positive regulation of cell migration & 4 & $5.34 \times 10^{-4}$ \\
\hline GO TERM_BP_DIRECT & GO:0038083 peptidyl-tyrosine autophosphorylation & 3 & $5.70 \times 10^{-4}$ \\
\hline GO TERM_BP_DIRECT & GO:0008284 positive regulation of cell proliferation & 5 & $6.27 \times 10^{-4}$ \\
\hline
\end{tabular}

B, VAT

\begin{tabular}{|c|c|c|c|}
\hline Category & Term & Count & P-value \\
\hline KEGG_PATHWAY & hsa04640:Hematopoietic cell lineage & 3 & $2.29 \times 10^{-3}$ \\
\hline GOTERM_BP_DIRECT & GO:0006955 immune response & 3 & $1.59 \times 10^{-2}$ \\
\hline KEGG_PATHWAY & hsa04060:Cytokine-cytokine receptor interaction & 3 & $1.70 \times 10^{-2}$ \\
\hline GOTERM_CC_DIRECT & GO:0005615 extracellular space & 4 & $1.70 \times 10^{-2}$ \\
\hline GOTERM_BP_DIRECT & GO:0030838 positive regulation of actin filament polymerization & 2 & $2.12 \times 10^{-2}$ \\
\hline GOTERM_MF_DIRECT & $\begin{array}{l}\text { GO:0016705 oxidoreductase activity, acting on paired donors, with } \\
\text { incorporation or reduction of molecular oxygen }\end{array}$ & 2 & $2.67 \times 10^{-2}$ \\
\hline GOTERM_BP_DIRECT & GO:0043547 positive regulation of GTPase activity & 3 & $2.77 \times 10^{-2}$ \\
\hline GOTERM_BP_DIRECT & GO:0014068 positive regulation of phosphatidylinositol 3-kinase signaling & 2 & $3.06 \times 10^{-2}$ \\
\hline GOTERM_CC_DIRECT & GO:0031090 organelle membrane & 2 & $3.76 \times 10^{-2}$ \\
\hline GOTERM_CC_DIRECT & GO:0016021 integral component of membrane & 6 & $4.59 \times 10^{-2}$ \\
\hline
\end{tabular}

GCN, gene co-expression network; SAT, subcutaneous adipose tissue; VAT, visceral adipose tissue; GO, Gene Ontology; KEGG, Kyoto Encyclopedia of Genes and Genomes; BP, biological process; MF, molecular function; CC, cellular components.

Table III. Summary of depot-specific expression quantitative trait loci that regulate the expression of LGI family member 3.

\begin{tabular}{|c|c|c|c|c|c|}
\hline Variant ID & SNV ID & P-value & NES & MAF & Tissue \\
\hline chr8_21801331_T_G_b38 & rs373132699 & $2.9 \times 10^{-5}$ & -0.9 & 0.0018 & SAT \\
\hline chr8_23149508_G_A_b38 & rs 13256716 & $1.7 \times 10^{-5}$ & 0.18 & 0.2514 & SAT \\
\hline chr8_23149665_G_A_b38 & rs 13257094 & $4.7 \times 10^{-5}$ & 0.17 & 0.2618 & SAT \\
\hline chr8_22361155_C_T_b38 & rs56251182 & $6.4 \times 10^{-6}$ & -0.79 & 0.0174 & VAT (Omentum) \\
\hline
\end{tabular}

Nominal P-values were generated by the linear regression model between genotype and expression (https://gtexportal.org/home/documentationPage). SNV, single nucleotide variations; NES, normalized effect size; MAF, minor allele frequency; SAT, subcutaneous adipose tissue; VAT, visceral adipose tissue.

and prognostic roles in obesity-associated metabolic diseases through macrophage polarization in VATs. The limitations of the present study were that mouse epididymal adipose tissues and 3T3-L1 cells were used for array analyses and GCN and eQTL databases for integrative analysis. The present results warrant further studies to validate the predominant proinflammatory role of LGI3 in VAT via M1 macrophage polarization using VAT from obese animals and humans.

In conclusion, the present study provided an integrative insight into LGI3-regulated gene products and the association 
of their protein-protein interaction and co-expression networks in adipose tissues with common and differential biological processes in SATs and VATs. It is hypothesized from these data that LGI3 may serve a homeostatic and pathological role in adipose tissues in a depot-specific manner.

\section{Acknowledgements}

Not applicable.

\section{Funding}

This work was supported by a National Research Foundation of Korea (NRF) grant funded by the Korean government Ministry of Science and ICT (grant. no. 2018R1D1A1A09082440).

\section{Availability of data and materials}

The datasets used and/or analyzed during the current study are not deposited in public repositories due to pending patent but are available from the corresponding author on reasonable request.

\section{Authors' contributions}

HYY conceived and designed the study, performed the data analysis and wrote the manuscript. HAK performed the experiments and data analysis. KJB contributed to the analysis and interpretation of data. HYY, HAK and KJB confirm the authenticity of all the raw data. All the authors read and approved the final manuscript.

\section{Ethics approval and consent to participate}

Not applicable.

\section{Patient consent for publication}

Not applicable.

\section{Competing interests}

The datasets used and/or analyzed during the current study are partially related with pending patent (HYY, Korean patent application reference no. 10-2018-0060762. 2018.5.28). The authors declare that they have no other competing interests.

\section{References}

1. Lee SE, Lee AY, Park WJ, Jun DH, Kwon NS, Baek KJ, Kim YG and Yun HY: Mouse LGI3 gene: Expression in brain and promoter analysis. Gene 372: 8-17, 2006.

2. Park WJ, Lee SE, Kwon NS, Baek KJ, Kim DS and Yun HY: Leucine-rich glioma inactivated 3 associates with syntaxin 1. Neurosci Lett 444: 240-244, 2008.

3. Park WJ, Lim YY, Kwon NS, Baek KJ, Kim DS and Yun HY: Leucine-rich glioma inactivated 3 induces neurite outgrowth through Akt and focal adhesion kinase. Neurochem Res 35: 789-796, 2010

4. Lee SH, Jeong YM, Kim SY, Jeong HS, Park KC, Baek KJ, Kwon NS, Yun HY and Kim DS: Ultraviolet B-induced LGI3 secretion protects human keratinocytes. Exp Dermatol 21: 716-718, 2012.
5. Jeong YM, Park WJ, Kim MK, Baek KJ, Kwon NS, Yun HY and Kim DS: Leucine-rich glioma inactivated 3 promotes HaCaT keratinocyte migration. Wound Repair Regen 21: 634-640, 2013.

6. Kim IW, Jeong HS, Kwon NS, Baek KJ, Yun HY and Kim DS: LGI3 promotes human keratinocyte differentiation via the Akt pathway. Exp Dermatol 27: 1224-1229, 2018.

7. Kim US, Park JW, Park ES, Bang JS, Jung TW, Kim DS, Abd El-Aty AM, Lee JH and Jeong JH: The suppressive effect of leucine-rich glioma inactivated 3 (LGI3) peptide on impaired skin barrier function in a murine model atopic dermatitis. Pharmaceutics 12: 750, 2020.

8. Lee SH, Kwon NS, Baek KJ, Yun HY and Kim DS: LGI3 is secreted and binds to ADAM22 via TRIF-dependent $\mathrm{NF}-\kappa \mathrm{B}$ pathway in response to LPS in human keratinocytes. Cytokine 126: 154872, 2020.

9. Jeong HS, Jeong YM, Kim J, Lee SH, Choi HR, Park KC, Kim BJ, Baek KJ, Kwon NS, Yun HY and Kim DS: Leucine-rich glioma inactivated 3 is a melanogenic cytokine in human skin. Exp Dermatol 23: 600-602, 2014.

10. Kwon NS, Baek KJ, Kim DS and Yun HY: Leucine-rich glioma inactivated 3: Integrative analyses reveal its potential prognostic role in cancer. Mol Med Rep 17: 3993-4002, 2018.

11. Kwon NS, Kim DS and Yun HY: Leucine-rich glioma inactivated 3: Integrative analyses support its prognostic role in glioma. Onco Targets Ther 10: 2721-2728, 2017.

12. Kim HA, Kwon NS, Baek KJ, Kim DS and Yun HY: Leucine-rich glioma inactivated 3: Integrative analyses support its role in the cytokine network. Int J Mol Med 40: 251-259, 2017.

13. Kim DS, Kwon NS and Yun HY: Leucine rich repeat LGI family member 3: Integrative analyses reveal its prognostic association with non-small cell lung cancer. Oncol Lett 18: 3388-3398, 2019.

14. Kim HA, Park WJ, Jeong HS, Lee HE, Lee SH, Kwon NS, Baek KJ, Kim DS and Yun HY: Leucine-rich glioma inactivated 3 regulates adipogenesis through ADAM23. Biochim Biophys Acta 1821: 914-922, 2012.

15. Kim HA, Kwon NS, Baek KJ, Kim DS and Yun HY: Leucine-rich glioma inactivated 3 associates negatively with adiponectin. Cytokine 62: 206-209, 2013.

16. Kim HA, Kwon NS, Baek KJ, Kim DS and Yun HY: Leucine-rich glioma inactivated 3 and tumor necrosis factor- $\alpha$ regulate mutually through NF- $\kappa$ B. Cytokine 72: 220-223, 2015.

17. Animal research: Reporting of in vivo experiments (ARRIVE) guidelines. Available from: https://arriveguidelines.org.

18. Szklarczyk D, Franceschini A, Wyder S, Forslund K, Heller D, Huerta-Cepas J, Simonovic M, Roth A, Santos A, Tsafou KP, et al: STRING v10: Protein-protein interaction networks, integrated over the tree of life. Nucleic Acids Res 43: D447-D452, 2015.

19. Lopes CT, Franz M, Kazi F, Donaldson SL, Morris Q and Bader GD: Cytoscape web: An interactive web-based network browser. Bioinformatics 26: 2347-2348, 2010.

20. Alvarez-Ponce D, Feyertag F and Chakraborty S: Position matters: Network centrality considerably impacts rates of protein evolution in the human protein-protein interaction network. Genome Biol Evol 9: 1742-1756, 2017.

21. Huang dW, Sherman BT and Lempicki RA: Systematic and integrative analysis of large gene lists using DAVID bioinformatics resources. Nat Protoc 4: 44-57, 2009.

22. Lee S, Zhang C, Liu Z, Klevstig M, Mukhopadhyay B, Bergentall M, Cinar R, Stahlman M, Sikanic N, Park JK, et al: Network analyses identify liver-specific targets for treating liver diseases. Mol Syst Biol 13: 938, 2017.

23. Pratt D, Chen J, Welker D, Rivas R, Pillich R, Rynkov V, Ono K, Miello C, Hicks L, Szalma S, et al: NDEx, the network data exchange. Cell Syst 1: 302-305, 2015.

24. Maere S, Heymans K and Kuiper M: BiNGO: A cytoscape plugin to assess overrepresentation of gene ontology categories in biological networks. Bioinformatics 21: 3448-3449, 2005.

25. Thomas-Chollier M, Hufton A, Heinig M, O'Keeffe S, Masri NE, Roider HG, Manke T and Vingron M: Transcription factor binding predictions using TRAP for the analysis of ChIP-seq data and regulatory SNPs. Nat Protoc 6: 1860-1869, 2011.

26. GTEx Consortium: The genotype-tissue expression (GTEx) project. Nat Genet 45: 580-585, 2013.

27. Stuart JM, Segal E, Koller D and Kim SK: A gene-coexpression network for global discovery of conserved genetic modules. Science 302: 249-255, 2003.

28. Zhang Q, Lenardo MJ and Baltimore D: 30 years of NF- $\kappa$ B: A blossoming of relevance to human pathobiology. Cell 168: 37-57, 2017. 
29. Sabir JS, El Omri A, Shaik NA, Banaganapalli B, Al-Shaeri MA Alkenani NA, Hajrah NH, Awan ZA, Zrelli H, Elango R and Khan M: Identification of key regulatory genes connected to $\mathrm{NF}-\kappa \mathrm{B}$ family of proteins in visceral adipose tissues using gene expression and weighted protein interaction network. PLoS One 14: e0214337, 2019.

30. Verbisck NV, Costa ET, Costa FF, Cavalher FP, Costa MD, Muras A, Paixão VA, Moura R, Granato MF, Ierardi DF, et al: ADAM23 negatively modulates alpha(v)beta(3) integrin activation during metastasis. Cancer Res 69: 5546-5552, 2009.

31. Antonov AS, Antonova GN, Munn DH, Mivechi N, Lucas R, Catravas JD and Verin AD: $\alpha \mathrm{V} \beta 3$ integrin regulates macrophage inflammatory responses via PI3 kinase/Akt-dependent NF- $\kappa \mathrm{B}$ activation. J Cell Physiol 226: 469-476, 2011.

32. Huang XF and Chen JZ: Obesity, the PI3K/Akt signal pathway and colon cancer. Obes Rev 10: 610-616, 2009.

33. Mauer J, Chaurasia B, Goldau J, Vogt MC, Ruud J, Nguyen KD, Theurich S, Hausen AC, Schmitz J, Brönneke HS, et al: Signaling by IL- 6 promotes alternative activation of macrophages to limit endotoxemia and obesity-associated resistance to insulin. Nat Immunol 15: 423-430, 2014.

34. Yokoyama M, Okada S, Nakagomi A, Moriya J, Shimizu I, Nojima A, Yoshida Y, Ichimiya H, Kamimura N, Kobayashi Y, et al: Inhibition of endothelial p53 improves metabolic abnormalities related to dietary obesity. Cell Rep 7: 1691-1703, 2014.

35. Adachi H, Kurachi H, Homma H, Adachi K, Imai T, Sakata M, Matsuzawa Y and Miyake A: Involvement of epidermal growth factor in inducing adiposity of age female mice. J Endocrinol 146 381-393, 1995.

36. Hotamisligil GS, Shargill NS and Spiegelman BM: Adipose expression of tumor necrosis factor-alpha: Direct role in obesity-linked insulin resistance. Science 259: 87-91, 1993.

37. Lukjanenko L, Jung MJ, Hegde N, Perruisseau-Carrier C, Migliavacca E, Rozo M, Karaz S, Jacot G, Schmidt M, Li L, et al: Loss of fibronectin from the aged stem cell niche affects the regenerative capacity of skeletal muscle in mice. Nat Med 22 897-905, 2016

38. Jager J, Corcelle V, Grémeaux T, Laurent K, Waget A, Pagès G Binétruy B, Le Marchand-Brustel Y, Burcelin R, Bost F and Tanti JF: Deficiency in the extracellular signal-regulated kinase 1 (ERK1) protects leptin-deficient mice from insulin resistance without affecting obesity. Diabetologia 54: 180-189, 2011.

39. Donohoe F, Wilkinson M, Baxter E and Brennan DJ: Mitogenactivated protein kinase (MAPK) and obesity-related cancer. Int J Mol Sci 21: 1241, 2020.

40. Pal A, Barber TM, Van de Bunt M, Rudge SA, Zhang Q, Lachlan KL, Cooper NS, Linden H, Levy JC, Wakelam MJ, et al: PTEN mutations as a cause of constitutive insulin sensitivity and obesity. N Engl J Med 367: 1002-1011, 2012.

41. Berryman DE, Glad CA, List EO and Johannsson G: The GH/IGF-1 axis in obesity: Pathophysiology and therapeutic considerations. Nat Rev Endocrinol 9: 346-356, 2013.

42. Zhang X, Xu A, Chung SK, Cresser JH, Sweeney G, Wong RL, Lin A and Lam KS: Selective inactivation of c-Jun NH2-terminal kinase in adipose tissue protects against diet-induced obesity and improves insulin sensitivity in both liver and skeletal muscle in mice. Diabetes 60: 486-495, 2011.

43. Rull A, Camps J, Alonso-Villaverde C and Joven J: Insulin resistance, inflammation, and obesity: Role of monocyte chemoattractant protein-1 (or CCL2) in the regulation of metabolism. Mediators Inflamm 2010: 326580, 2010.

44. Cai H, Dong LQ and Liu F: Recent advances in adipose mTOR signaling and function: Therapeutic prospects. Trends Pharmacol Sci 37: 303-317, 2016.

45. Chen M, Lu P, Ma Q, Cao Y, Chen N, Li W, Zhao S, Chen B, Shi J, Sun Y, et al: CTNNB1/ß-catenin dysfunction contributes to adiposity by regulating the cross-talk of mature adipocytes and preadipocytes. Sci Adv 6: eaax9605, 2020.

46. Matesanz N, Nikolic I, Leiva M, Pulgarin-Alfaro M, Santamans AM, Bernardo E, Mora A, Herrera-Melle L, Rodriguez E, Beiroa D, et al: p38 a blocks brown adipose tissue thermogenesis through p38 $\delta$ inhibition. PLoS Biol 16: e2004455, 2018.

47. Fatima LA, Campello RS, Santos RS, Freitas HS, Frank AP, Machado UF and Clegg DJ: Estrogen receptor 1 (ESR1) regulates VEGFA in adipose tissue. Sci Rep 7: 16716, 2017.

48. Kubota T, Kubota N and Kadowaki T: Imbalanced insulin actions in obesity and type 2 diabetes: Key mouse models of insulin signaling pathway. Cell Metab 25: 797-810, 2017.
49. Ray A: Tumor-linked HER2 expression: Association with obesity and lipid-related microenvironment. Horm Mol Biol Clin Investig 32, 2017.

50. Huang X, Liu G, Guo J and Su Z: The PI3K/AKT pathway in obesity and type 2 diabetes. Int J Biol Sci 14: 1483-1496, 2018.

51. Lefterova MI, Haakonsson AK, Lazar MA and Mandrup S: PPAR $\gamma$ and the global map of adipogenesis and beyond. Trends Endocrinol Metab 25: 293-302, 2014.

52. Antonopoulos AS, Margaritis M, Coutinho P, Shirodaria C, Psarros C, Herdman L, Sanna F, De Silva R, Petrou M, Sayeed R, et al: Adiponectin as a link between type 2 diabetes and vascular NADPH oxidase activity in the human arterial wall: The regulatory role of perivascular adipose tissue. Diabetes 64: 2207-2219, 2015

53. Du J, Fan LM, Mai A and Li JM: Crucial roles of Nox2-derived oxidative stress in deteriorating the function of insulin receptors and endothelium in dietary obesity of middle-aged mice. Br J Pharmacol 170: 1064-1077, 2013.

54. Fernandez-Twinn DS, Blackmore HL, Siggens L, Giussani DA, Cross CM, Foo R and Ozanne SE: The programming of cardiac hypertrophy in the offspring by maternal obesity is associated with hyperinsulinemia, AKT, ERK, and mTOR activation. Endocrinology 153: 5961-5971, 2012

55. Kanda H, Tateya S, Tamori Y, Kotani K, Hiasa K, Kitazawa R, Kitazawa S, Miyachi H, Maeda S, Egashira K and Kasuga M: $\mathrm{MCP}-1$ contributes to macrophage infiltration into adipose tissue, insulin resistance, and hepatic steatosis in obesity. J Clin Invest 116: 1494-1505, 2006.

56. Kapur S, Marcotte B and Marette A: Mechanism of adipose tissue iNOS induction in endotoxemia. Am J Physiol 276: E635-E641, 1999.

57. Pietiläinen KH, Kannisto K, Korsheninnikova E, Rissanen A, Kaprio J, Ehrenborg E, Hamsten A and Yki-Järvinen H: Acquired obesity increases CD68 and tumor necrosis factor-alpha and decreases adiponectin gene expression in adipose tissue: A study in monozygotic twins. J Clin Endocrinol Metab 91: 2776-2781, 2006.

58. Ronis MJ, Sharma N, Vantrease J, Borengasser SJ, Ferguson M, Mercer KE, Cleves MA, Gomez-Acevedo H and Badger TM: Female mice lacking p47phox have altered adipose tissue gene expression and are protected against high fat-induced obesity. Physiol Genomics 45: 351-366, 2013.

59. Sindhu S, Thomas R, Shihab P, Sriraman D, Behbehani K and Ahmad R: Obesity is a positive modulator of IL-6R and IL-6 expression in the subcutaneous adipose tissue: Significance for metabolic inflammation. PLoS One 10: e0133494, 2015.

60. Uchida K, Satoh M, Inoue G, Onuma K, Miyagi M, Iwabuchi K and Takaso M: CD11c(+) macrophages and levels of TNF- $\alpha$ and MMP-3 are increased in synovial and adipose tissues of osteoarthritic mice with hyperlipidaemia. Clin Exp Immunol 180 551-559, 2015.

61. Weisberg SP, McCann D, Desai M, Rosenbaum M, Leibel RL and Ferrante AW Jr: Obesity is associated with macrophage accumulation in adipose tissue. J Clin Invest 112: 1796-1808, 2003.

62. Min JL, Nicholson G, Halgrimsdottir I, Almstrup K, Petri A, Barrett A, Travers M, Rayner NW, Mägi R, Pettersson FH, et al: Coexpression network analysis in abdominal and gluteal adipose tissue reveals regulatory genetic loci for metabolic syndrome and related phenotypes. PLoS Genet 8: e1002505, 2012.

63. Skinkyte-Juskiene R, Kogelman LJ and Kadarmideen HN: Transcription factor co-expression networks of adipose RNA-seq data reveal regulatory mechanisms of obesity. Curr Genomics 19: 289-299, 2018.

64. Lumeng CN, DelProposto JB, Westcott DJ and Saltiel AR: Phenotypic switching of adipose tissue macrophages with obesity is generated by spatiotemporal differences in macrophage subtypes. Diabetes 57: 3239-3246, 2008.

65. Mosser DM and Edwards JP: Exploring the full spectrum of macrophage activation. Nat Rev Immunol 8: 958-969, 2008

66. Appari M, Channon KM and McNeill E: Metabolic regulation of adipose tissue macrophage function in obesity and diabetes. Antioxid Redox Signal 29: 297-312, 2018.

67. Morris DL, Singer K and Lumeng CN: Adipose tissue macrophages: Phenotypic plasticity and diversity in lean and obese states. Curr Opin Clin Nutr Metab Care 14: 341-346, 2011.

68. Mathis D: Immunological goings-on in visceral adipose tissue Cell Metab 17: 851-859, 2013.

This work is licensed under a Creative Commons Attribution-NonCommercial-NoDerivatives 4.0 International (CC BY-NC-ND 4.0) License. 